\title{
Cryptanalysis of the TTM Cryptosystem
}

\author{
Louis Goubin and Nicolas T. Courtois \\ Bull CP8 \\ 68 route de Versailles - BP45 \\ 78431 Louveciennes Cedex \\ France \\ Louis.Goubin@bull.net, courtois@minrank.org
}

\begin{abstract}
In 1985 Fell and Diffie proposed constructing trapdoor functions with multivariate equations [11. They used several sequentially solved stages that combine into a triangular system we call $\mathrm{T}$. In the present paper, we study a more general family of TPM (for "Triangle Plus Minus") schemes: a triangular construction mixed with some $u$ random polynomials and with some $r$ of the beginning equations removed. We go beyond all previous attacks proposed on such cryptosystems using a low degree component of the inverse function. The cryptanalysis of TPM is reduced to a simple linear algebra problem called MinRank(r): Find a linear combination of given matrices that has a small rank $r$. We introduce a new attack for MinRank called 'Kernel Attack' that works for $q^{r}$ small. We explain that TPM schemes can be used in encryption only if $q^{r}$ is small and therefore they are not secure.

As an application, we showed that the TTM cryptosystem proposed by T.T. Moh at CrypTec'99 [15/16 reduces to MinRank(2). Thus, though the cleartext size is 512 bits, we break it in $\mathcal{O}\left(2^{52}\right)$. The particular TTM of 15 16 can be broken in $\mathcal{O}\left(2^{28}\right)$ due additional weaknesses, and we needed only few minutes to solve the challenge TTM 2.1. from the website of the TTM selling company, US Data Security.

We also studied TPM in signature, possible only if $q^{u}$ small. It is equally insecure: the 'Degeneracy Attack' we introduce runs in $q^{u}$. polynomial.
\end{abstract}

\section{Introduction}

The current research effort in practical public key cryptography introduced by Rivest, Shamir and Adleman, with univariate polynomials over $\mathbb{Z}_{N}$, is following two paths. The first is considering more complex groups, e.g. elliptic curves. The second is considering multivariate equations. Though many proposed schemes are being broken, some remain unbroken even for the simplest groups like $\mathbb{Z}_{2}$.

One of the paradigms for constructing multivariate trapdoor cryptosystems is the triangular construction, proposed initially in an iterated form by Fell and Diffie (1985). It uses equations that involve $1,2, \ldots, n$ variables and are solved sequentially. The special form of the equations is hidden by two linear transformations on inputs (variables) and outputs (equations). We call $\mathrm{T}$ this triangular construction. Let TPM (T Plus-Minus) be T with added final $u$ random (fullsize) quadratic polynomials, and with $r$ of the beginning equations removed.

T. Okamoto (Ed.): ASIACRYPT 2000, LNCS 1976, pp. 44-57, 2000.

(C) Springer-Verlag Berlin Heidelberg 2000 
The cryptosystem TTM, proposed by T.T. Moh at CrypTec'99 is in spite of an apparent complexity, shown in 2.4 to be a subcase of TPM. The initially proposed scheme is very weak due to linear dependencies and in section 4.2 , we present the solution (plaintext) to the TTM 2.1 challenge proposed by the company US Data Security, which is currently selling implementations of TTM. After this, we focus on breaking more general TPM schemes.

The general strategy to recover the secret key of TPM/TTM systems is presented in 3 . It requires finding a linear combination of public equations that depends only of a subspace of variables. This gives a simple linear algebra problem called MinRank: Let us consider some $n \times n$ matrices over $\operatorname{GF}(q): M_{1}, \ldots, M_{t}$. We need to find a linear combination $M$ of the $M_{i}$ that has a small rank $r<n$. The name of MinRank has apparently been used first in the paper [19] that shows that MinRank is NP-complete. However the MinRank instances in TPM/TTM use very small $r$, e.g. the T.T. Moh's proposal from [16] gives $r=2$. We note that the powerful idea of using a small rank goes back to the cryptanalysis of Shamir birational scheme [20 by Coppersmith, Stern and Vaudenay [6.7], and appears also in the Shamir-Kipnis attack on HFE [14] proposed by Patarin [17.

In 2.2 we explain how to use the TPM schemes in encryption which is possible only if $q^{r}$ is small. However, in the section 5 we present an attack that works precisely when $q^{r}$ is small, based on the small co-dimension of the kernel of the unknown matrix $M$. This 'Kernel attack' breaks in approximately $2^{52}$ a cryptosystem with 512 bit cleartexts.

Similarly in 2.2 we explain how to use the TPM schemes in signature; possible only with $q^{u}$ not too big. Then in section 6 we introduce the 'Degeneracy attack' on TPM based on iterative searching of degenerate polynomials. It works precisely when $q^{u}$ is small and the signature proposals of [15[16] are insecure.

\section{The TPM Family of Cryptosystems}

\subsection{General Description of TPM}

In the present section, we describe the general family $\operatorname{TPM}(n, u, r, K)$, with:

$-n, u, r$ integers such that $r \leq n$. We also systematically put $m=n+u-r$.

$-K=\operatorname{GF}(q)$ a finite field.

We first consider a function $\Psi: K^{n} \mapsto K^{n+u-r}$ such that $\left(y_{1}, \ldots, y_{n+u-r}\right)=$ $\Psi\left(x_{1}, \ldots, x_{n}\right)$ is defined by the following system of equations:

$$
\left\{\begin{array}{rlrl}
y_{1} & =x_{1}+ & g_{1}( & \left.x_{n-r+1}, \ldots, x_{n}\right) \\
y_{2} & =x_{2}+ & g_{2}\left(x_{1} ;\right. & \\
y_{3} & =x_{3}+ & g_{3}\left(x_{1}, x_{2} ;\right. & \\
\vdots & & & \left.x_{n-r+1}, \ldots, x_{n}\right) \\
y_{n-r} & =x_{n-r}+g_{n-r}\left(x_{1}, \ldots, x_{n-r-1}\right) \\
y_{n-r+1} & = & & g_{n-r+1}\left(x_{1}, \ldots, x_{n}\right) \\
\vdots & & & \\
y_{n-r+u} & = & & \\
& & &
\end{array}\right.
$$


with each $g_{i}(1 \leq i \leq n+u-r)$ being a randomly chosen quadratic polynomial.

\section{The Public Key}

The user selects a random invertible affine transformation $s: K^{n} \mapsto K^{n}$, and a random invertible affine transformation $t: K^{n+u-r} \mapsto K^{n+u-r}$. Let $F=t \circ \Psi \circ s$. By construction, if we denote $\left(y_{1}^{\prime}, \ldots, y_{n+u-r}^{\prime}\right)=F\left(x_{1}^{\prime}, \ldots, x_{n}^{\prime}\right)$, we obtain an explicit set $\left\{P_{1}, \ldots, P_{n+u-r}\right\}$ of $(n+u-r)$ quadratic polynomials in $n$ variables, such that:

$$
\left\{\begin{array}{l}
y_{1}^{\prime}=P_{1}\left(x_{1}^{\prime}, \ldots, x_{n}^{\prime}\right) \\
\quad \vdots \\
y_{n+u-r}^{\prime}=P_{n+u-r}\left(x_{1}^{\prime}, \ldots, x_{n}^{\prime}\right)
\end{array}\right.
$$

This set of $(n+u-r)$ quadratic polynomials constitute the public key of this $\operatorname{TPM}(n, u, r, K)$ cryptosystem. Its size is $\frac{1}{8}(n+u-r)(n+1)\left(\frac{n}{2}+1\right) \log _{2}(q)$ bytes.

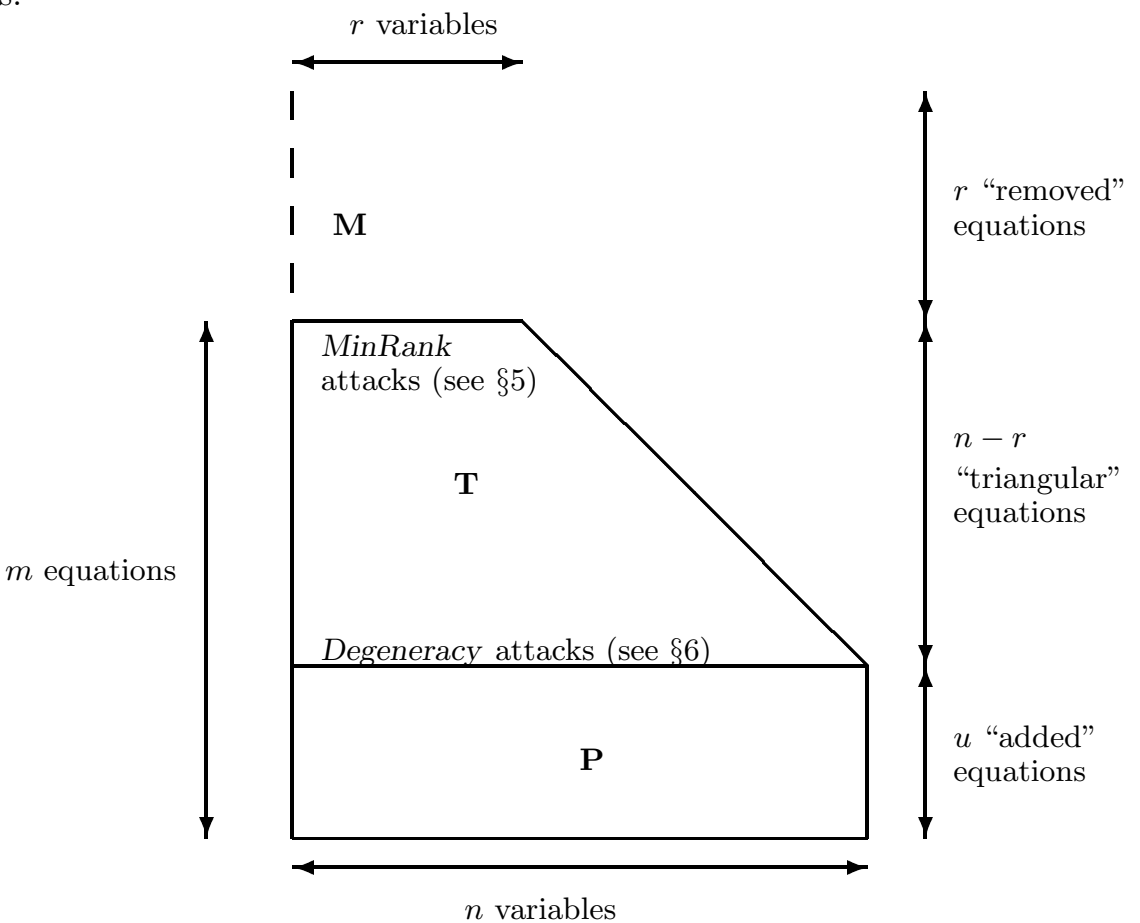

Fig. 1. General view of the TPM scheme - The two classes of attacks

\subsection{Encryption Protocol (when $u \geq r$ )}

\section{Encrypting a message}

Given a plaintext $\left(x_{1}^{\prime}, \ldots, x_{n}^{\prime}\right) \in K^{n}$, the sender computes $y_{i}^{\prime}=P_{i}\left(x_{1}^{\prime}, \ldots, x_{n}^{\prime}\right)$ for $1 \leq i \leq n+u-r-$ thanks to the public key - and sends the ciphertext $\left(y_{1}^{\prime}, \ldots, y_{n+u-r}^{\prime}\right) \in K^{n+u-r}$. 


\section{Decrypting a message}

Given a ciphertext $\left(y_{1}^{\prime}, \ldots, y_{n+u-r}^{\prime}\right) \in K^{n+u-r}$, the legitimate receiver recovers the plaintext by the following method.

- Compute $\left(y_{1}, \ldots, y_{n+u-r}\right)=t^{-1}\left(y_{1}^{\prime}, \ldots, y_{n+u-r}^{\prime}\right)$;

- Make an exhaustive search on the $r$-tuple $\left(x_{n-r+1}, \ldots, x_{n}\right) \in K^{r}$, until the $n$-tuple $\left(x_{1}, \ldots, x_{n}\right)$ obtained by $x_{i}=y_{i}-g_{i}\left(x_{1}, \ldots, x_{i-1} ; x_{n-r+1}, \ldots, x_{n}\right)$ (for $1 \leq i \leq n-r)$ satisfies the $u$ following equations $g_{i}\left(x_{1}, \ldots, x_{n}\right)=y_{i}$ (for $n-r+1 \leq i \leq n-r+u)$.

- For the obtained $\left(x_{1}, \ldots, x_{n}\right) n$-tuple, get $\left(x_{1}^{\prime}, \ldots, x_{n}^{\prime}\right)=s^{-1}\left(x_{1}, \ldots, x_{n}\right)$.

This decryption algorithm thus has a complexity essentially $\mathcal{O}\left(q^{r}\right)$. As a result, a $\operatorname{TPM}(n, u, r, K)$ cryptosystem can be practically used in encryption mode only under the assumption that $q^{r}$ is "small enough".

The condition $u \geq r$ insures that the probability of obtaining a collision is negligible, and thus that the ciphering function $F$ can be considered as an injection from $K^{n}$ into $K^{n+u-r}$.

When $r=u=0$, this kind of scheme has been considered and attacked by Fell and Diffie in [1] (in an iterated form) and by Patarin and Goubin in [18. All these attacks explore the fact that the inverse function if of low degree in some variables, whereas the present paper cryptanalyses much more general cases with $r \neq 0$ and $u \neq 0$.

\subsection{Signature Protocol (when $u \leq r$ )}

\section{Signing a message}

Given a message $M$, we suppose that $\left(y_{1}^{\prime}, \ldots, y_{n+u-r}^{\prime}\right)=h(M) \in K^{n+u-r}$, with $h$ being a (collision-free) hash function. To sign the message $M$, the legitimate user:

- computes $\left(y_{1}, \ldots, y_{n+u-r}\right)=t^{-1}\left(y_{1}^{\prime}, \ldots, y_{n+u-r}^{\prime}\right)$;

- chooses random $r$-tuples $\left(x_{n-r+1}, \ldots, x_{n}\right)$, until the $n$-tuple $\left(x_{1}, \ldots, x_{n}\right)$ obtained by $x_{i}=y_{i}-g_{i}\left(x_{1}, \ldots, x_{i-1} ; x_{n-r+1}, \ldots, x_{n}\right)$ (for $\left.1 \leq i \leq n-r\right)$ satisfies the $u$ following equations $g_{i}\left(x_{1}, \ldots, x_{n}\right)=y_{i}($ for $n-r+1 \leq i \leq n-r+u)$.

- for the obtained $\left(x_{1}, \ldots, x_{n}\right) n$-tuple, gets $\left(x_{1}^{\prime}, \ldots, x_{n}^{\prime}\right)=s^{-1}\left(x_{1}, \ldots, x_{n}\right)$.

This signature algorithm thus has a complexity essentially $\mathcal{O}\left(q^{u}\right)$. As a result, a $\operatorname{TPM}(n, u, r, K)$ cryptosystem can be practically used in signature mode only under the assumption that $q^{u}$ is "small enough".

The condition $u \leq r$ insures that the probability of finding no solution for $\left(x_{1}, \ldots, x_{n}\right)$ for the equation $\Psi\left(x_{1}, \ldots, x_{n}\right)=\left(y_{1}, \ldots, y_{n+u-r}\right)$ is negligible, and thus that the ciphering function $F$ can be viewed as an surjection from $K^{n}$ onto $K^{n+u-r}$.

We will describe in section [6 a general attack on this signature scheme, that is also applicable when $u$ is non-zero, with $q^{u}$ not too large. Therefore the signature proposed by T.T. Moh in [15,16] is insecure. 


\subsection{The TTM Encryption System}

In the present section, we recall the original description of the TTM cryptosystem, given by T.T. Moh in [15,16]. This definition of TTM is based on the concept of tame automorphisms. As we will see, TTM is a particular case of our general family TPM: it belongs to the family $\operatorname{TPM}(64,38,2, \mathrm{GF}(256))$.

\section{General Principle}

Let $K$ be a finite field (which will be supposed "small" in real applications). We first consider two bijections $\Phi_{2}$ and $\Phi_{3}$ from $K^{n+v}$ to $K^{n+v}$, with $\left(z_{1}, \ldots, z_{n+v}\right)=$ $\Phi_{2}\left(x_{1}, \ldots, x_{n+v}\right)$ and $\left(y_{1}, \ldots, y_{n+v}\right)=\Phi_{3}\left(z_{1}, \ldots, z_{n+v}\right)$ defined by the two following systems of equations :

$$
\Phi_{2}:\left\{\begin{array}{l}
z_{1}=x_{1} \\
z_{2}=x_{2}+f_{2}\left(x_{1}\right) \\
z_{3}=x_{3}+f_{3}\left(x_{1}, x_{2}\right) \\
\quad \vdots \\
z_{n}=x_{n}+f_{n}\left(x_{1}, \ldots, x_{n-1}\right) \\
z_{n+1}=x_{n+1}+f_{n+1}\left(x_{1}, \ldots, x_{n}\right) \\
\vdots \\
z_{n+v}=x_{n+v}+f_{n+v}\left(x_{1}, \ldots, x_{n+v-1}\right)
\end{array} \quad \Phi_{3}:\left\{\begin{array}{c}
y_{1}=z_{1}+P\left(z_{n+1}, \ldots, z_{n+v}\right) \\
y_{2}=z_{2}+Q\left(z_{n+1}, \ldots, z_{n+v}\right) \\
y_{3}=z_{3} \\
\vdots \\
y_{n+v}=z_{n+v}
\end{array}\right.\right.
$$

with $f_{2}, \ldots, f_{n+v}$ quadratic forms over $K$, and $P, Q$ two polynomials of degree eight over $K$.

$\Phi_{2}$ and $\Phi_{3}$ are both "tame automorphisms" (see [15]16] for a definition) and thus are one-to-one transformations. As a result, $\left(x_{1}, \ldots, x_{n+v}\right) \mapsto\left(y_{1}, \ldots, y_{n+v}\right)$ $=\Phi_{3} \circ \Phi_{2}\left(x_{1}, \ldots, x_{n+v}\right)$ is also one-to-one and can be described by the following system of equations:

$$
\left\{\begin{array}{l}
y_{1}=x_{1}+P\left(x_{n+1}+f_{n+1}\left(x_{1}, \ldots, x_{n}\right), \ldots, x_{n+v}+f_{n+v}\left(x_{1}, \ldots, x_{n+v-1}\right)\right) \\
y_{2}=x_{2}+f_{2}\left(x_{1}\right)+Q\left(x_{n+1}+f_{n+1}\left(x_{1}, . ., x_{n}\right), \ldots, x_{n+v}+f_{n+v}\left(x_{1}, . ., x_{n+v-1}\right)\right) \\
y_{3}=x_{3}+f_{3}\left(x_{1}, x_{2}\right) \\
\quad \vdots \\
y_{n}=x_{n}+f_{n}\left(x_{1}, \ldots, x_{n-1}\right) \\
y_{n+1}=x_{n+1}+f_{n+1}\left(x_{1}, \ldots, x_{n}\right) \\
\quad \vdots \\
y_{n+v}=x_{n+v}+f_{n+v}\left(x_{1}, \ldots, x_{n+v-1}\right)
\end{array}\right.
$$

T.T. Moh found a clever way of choosing $P, Q$ and $f_{i}$ such that $y_{1}$ and $y_{2}$ both become quadratic functions of $x_{1}, \ldots, x_{n}$ when we set $x_{n+1}=\ldots=x_{n+v}=0$.

\section{Actual Parameters}

This paragraph is given in the appendix. T.T. Moh chooses $n=64, v=36$ and $K=\mathrm{GF}(256)$. As a result, TTM belongs to $\mathrm{TPM}(64,38,2, \mathrm{GF}(256))$. Applying the formula of section 2.1, the size of the public keys is $214.5 \mathrm{Ko}$. 


\section{General Strategy of TPM Attacks}

In the present section, we describe a general strategy to attack a cryptosystem of the TPM Family when $r$ is "small". It will amount to solving the MinRank problem. As a result TTM, that is a $\operatorname{TPM}(64,38,2, \operatorname{GF}(256))$ will be broken.

\subsection{The MinRank Problem}

Let $r$ be an integer and $K$ a field. We denote by $\operatorname{MinRank}(r)$ the following problem: given a set $\left\{M_{1}, \ldots, M_{m}\right\}$ of $n \times n$ matrices whose coefficients lie in $K$, find at least one $m$-tuple $\left(\lambda_{1}, \ldots, \lambda_{m}\right) \in K^{m}$ such that $\operatorname{Rank}\left(\sum_{i=1}^{m} \lambda_{i} M_{i}\right) \leq r$.

The (even more) general MinRank problem has been first defined and studied by Shallit, Frandsen and Buss in [19]. It generalizes the "Rank Distance Coding" problem by Gabidulin 12], studied also in [3.22]), which itself generalizes the "Minimal Weight" problem of error correcting codes (see [1/21213]). In the Shamir-Kipnis attack on the Patarin's HFE cryptosystem [14,17], the authors used an instance of $\operatorname{MinRank}(r)$ with $r=\left\lceil\log _{q} n\right\rceil+1$ and therefore their attack is not polynomial. In the present paper $r$ is a small constant, e.g. 2. We note that the idea of finding small ranks has first been used by Coppersmith, Stern and Vaudenay in [6:7] for breaking Shamir's birational scheme [20].

Recently Courtois proposed a new zero-knowledge scheme based on MinRank [109]. Though in the present paper only two algorithms for MinRank are introduced, another two can be found in 9 .

\subsection{Complexity of MinRank}

The general MinRank problem has been proven to be NP-complete by Shallit, Frandsen and Buss (see 19]). More precisely, they prove that MinRank $(r)$ NPcomplete when $r=n-1$ (this corresponds to the problem of finding a linear combination of $M_{1}, \ldots, M_{m}$ that is singular). The principle of their proof consists in writing any set of multivariate equations as an instance of MinRank. It can be used in the same way to extend their result to the cases $r=n-2, r=n-3$, $\ldots$ and even $r=n^{\alpha}$ (when $\alpha>0$ is fixed). However, MinRank is not hard when $r$ gets smaller, indeed, in 5 we will introduce an expected polynomial time algorithm to solve the MinRank for any fixed $r$.

\subsection{Strategy of Attack}

We recall that $m=n+u-r$. We suppose $m \leq 2 n$, as an encryption function with expansion rate $>2$ is unacceptable. Moreover, if $m>\mathcal{O}(n)$, the cryptosystem is expected to be broken by Gröbner bases [8].

In each equation $y_{i}=x_{i}+g_{i}\left(x_{1}, \ldots, x_{i-1} ; x_{n-r+1}, \ldots, x_{n}\right)(1 \leq i \leq n-r)$, the homogeneous part is given by ${ }^{\mathrm{t}} X A_{i} X$, with ${ }^{\mathrm{t}} X=\left(x_{1}, \ldots, x_{n}\right), A_{i}$ being a (secret) matrix. Similarly, in each public equation $y_{i}^{\prime}=P_{i}\left(x_{1}^{\prime}, \ldots, x_{n}^{\prime}\right)$ is given by ${ }^{\mathrm{t}} X^{\prime} M_{i} X^{\prime}$, with ${ }^{\mathrm{t}} X^{\prime}=\left(x_{1}^{\prime}, \ldots, x_{n}^{\prime}\right), M_{i}$ being a (public) matrix. 
The fact that $\left(x_{1}, \ldots, x_{n}\right)=s\left(x_{1}^{\prime}, \ldots, x_{n}^{\prime}\right)$ and $\left(y_{1}^{\prime}, \ldots, y_{m}^{\prime}\right)=t\left(y_{1}, \ldots, y_{m}\right)$ implies that there exist an invertible $n \times n$ matrix $S$ and an invertible $m \times m$ matrix $T$ such that:

$$
\left(\begin{array}{c}
{ }^{\mathrm{t}}\left(S X^{\prime}\right) A_{1}\left(S X^{\prime}\right) \\
\vdots \\
{ }^{\mathrm{t}}\left(S X^{\prime}\right) A_{m}\left(S X^{\prime}\right)
\end{array}\right)=T^{-1}\left(\begin{array}{c}
{ }^{\mathrm{t}} X^{\prime} M_{1} X^{\prime} \\
\vdots \\
{ }^{\mathrm{t}} X^{\prime} M_{m} X^{\prime}
\end{array}\right) .
$$

Let $T^{-1}=\left(t_{i j}\right)_{1 \leq i, j \leq m}$. We thus have, for any $X^{\prime}$ :

$$
{ }^{\mathrm{t}} X^{\prime}\left({ }^{\mathrm{t}} S A_{i} S\right) X^{\prime}={ }^{\mathrm{t}} X^{\prime}\left(\sum_{j=1}^{m} t_{i j} M_{j}\right) X^{\prime}
$$

so that:

$$
\forall i, 1 \leq i \leq m, \quad \sum_{j=1}^{m} t_{i j} M_{j}={ }^{\mathrm{t}} S A_{i} S .
$$

From the construction of $\operatorname{TPM}(n, u, r, K)$, we have $\operatorname{Rank}\left(A_{1}\right) \leq r$. Since $S$ is an invertible matrix, we have $\operatorname{Rank}\left(A_{1}\right)=\operatorname{Rank}\left({ }^{\mathrm{t}} S A_{1} S\right)$ and thus Rank $\left(\sum_{j=1}^{m} t_{1 j} M_{j}\right) \leq r$, that is precisely an instance of $\operatorname{MinRank}(r)$.

Suppose we are able to find (at least) one $m$-tuple $\left(\lambda_{1}, \ldots, \lambda_{m}\right)$ such that $\operatorname{Rank}\left(\sum_{j=1}^{m} \lambda_{j} M_{j}\right) \leq r$. With a good probability, we can suppose that:

$$
\sum_{j=1}^{m} \lambda_{j} M_{j}=\mu^{\mathrm{t}} S A_{1} S \quad\left(\mu \in K^{*}\right) .
$$

Then we deduce the vector spaces $V_{0}=S^{-1}\left(K^{n-r} \times\{0\}^{r}\right)$ (corresponding to $\left.x_{n-r+1}=\ldots=x_{n}=0\right)$ and $W_{0}=S^{-1}\left(\{0\}^{n-r} \times K^{r}\right)$ (corresponding to $x_{1}=$ $\left.\ldots=x_{n-r}=0\right)$ by simply noticing that $V_{0}=\operatorname{Im}\left(\sum_{j=1}^{m} \lambda_{j} M_{j} A_{1}\right)$ and $W_{0}=$ $\operatorname{Ker}\left(\sum_{j=1}^{m} \lambda_{j} M_{j} A_{1}\right)$.

Once we have found $V_{0}$ and $W_{0}$, we can easily deduce the vector space $V_{1}=$ $S^{-1}\left(\{0\} \times K^{n-r-1} \times\{0\}^{r}\right)$ of dimension 1 (corresponding to $x_{1}=x_{n-r+1}=$ $\left.\ldots=x_{n}=0\right)$ and $W_{1}=S^{-1}\left(K \times\{0\}^{n-r-1} \times K^{r}\right)$ (corresponding to $x_{2}=$ $\left.\ldots=x_{n-r}=0\right)$ : we just look for coefficients $\alpha_{1}, \ldots, \alpha_{n}, \beta_{1}, \ldots, \beta_{m}$ such that the following equation:

$$
\sum_{j=1}^{m} \beta_{j} y_{j}^{\prime}=\sum_{i=1}^{n} \alpha_{i} x_{i}+\delta,
$$

holds for any element of $V_{0}$. This can be obtained by simple Gaussian reduction. We also obtain the $g_{2}$ quadratic function by Gaussian reduction. 
By repeating these steps, we obtain two sequences of vector spaces:

$$
\begin{gathered}
V_{0} \supseteq V_{1} \supseteq V_{2} \supseteq \ldots \supseteq V_{n-r-1} \\
W_{0} \subseteq W_{1} \subseteq W_{2} \subseteq \ldots \subseteq W_{n-r-1} .
\end{gathered}
$$

At the end, we have completely determined the secret transformations $s$ and $t$, together with the secret functions $g_{i}$. As a result, this algorithm completely breaks the TPM family of cryptosystems (we recovered the secret key).

\section{Special Case Attacks on TPM}

\subsection{The 'Linearity Attack' on TTM}

In this paragraph, we study the particular case of TTM, as described by T.T. Moh in 15 16]. In this case, we show that the $\operatorname{MinRank}(r)$ problem is easily solved, because of the particular structure of the $Q_{8}$ function used in $\Phi_{3}$.

\section{Description of the Attack}

In section 3.3, we proved that an attack can be successfully performed on TTM this cryptosystem, as soon as we can find out the vector spaces $V_{0}=$ $S^{-1}\left(\{0\}^{2} \times K^{62}\right)$ (corresponding to $\left.x_{1}=x_{2}=0\right)$ and $W_{0}=S^{-1}\left(K^{2} \times\{0\}^{62}\right)$ (corresponding to $x_{3}=\ldots=x_{64}=0$ ). At first sight, the equations giving $y_{1}$ and $y_{2}$ seem to be quadratic in $\left(x_{1}, \ldots, x_{64}\right)$. This leads a priori to an instance of $\operatorname{MinRank}(2)$.

However, note that the function $x \mapsto x^{2}$ is linear on $K=\mathrm{GF}(256)$, considered as a vector space of dimension 8 over $F=\mathrm{GF}(2)$. Therefore, considering the equations describing the (secret) $\Psi$ function of TTM 1 , if we choose a basis $\left(\omega_{1}, \ldots, \omega_{8}\right)$ of $K$ over $F$ and write $x_{i}=x_{i, 1} \omega_{1}+\ldots+x_{i, 8} \omega_{8}(1 \leq i \leq 64), y_{1}$ and $y_{2}$ become linear functions of $x_{1,1}, x_{1,2}, \ldots, x_{1,8}, \ldots, x_{64,1}, \ldots, x_{64,8}$. In terms of MinRank, this means that TTM leads to an instance of MinRank(0) for $8 n \times 8 n$ matrices (instead of an instance of $\operatorname{MinRank}(2)$ for $n \times n$ matrices). This leads to the following attack on TTM:

1. Let $x_{i}^{\prime}=x_{i, 1}^{\prime} \omega_{1}+\ldots+x_{i, 8}^{\prime} \omega_{8}(1 \leq i \leq 64)$. Rewrite each public equation $y_{i}^{\prime}=$ $P_{i}\left(x_{1}^{\prime}, \ldots, x_{64}^{\prime}\right)$ as $y_{i}^{\prime}=\tilde{P}_{i}\left(x_{1,1}^{\prime}, \ldots, x_{64,8}^{\prime}\right)$ (with $\tilde{P}_{i}$ a quadratic polynomial in $64 \times 8=512$ variables over $F=\mathrm{GF}(2))$.

2 . Find the vector space of the 612 -tuples $\left(\beta_{1}, \ldots, \beta_{100}, \alpha_{1,1}, \ldots, \alpha_{64,8}\right) \in K^{612}$ satisfying:

$$
\sum_{i=1}^{100} \beta_{i} y_{i}^{\prime}=\sum_{i=1}^{64} \sum_{j=1}^{8} \alpha_{i, j} x_{i, j}^{\prime} .
$$

This can be done by Gaussian reduction. We thus obtain the vector spaces $V_{0}$ and $W_{0}$ defined above.

3. The remaining part of the attack is exactly the same as in section 3.3

\footnotetext{
${ }^{1}$ See $(E)$ in the appendix, in which $t_{19}$ is a linear transformation.
} 


\section{Complexity of the Attack}

The main part of the algorithm consists in solving a system of linear equations on 612 variables, by Gaussian reduction. We thus obtain a complexity of approximately $2^{28}$ elementary operations to break TTM.

\subsection{Solution to the TTM 2.1 Challenge of US Data Security}

In 1997, US Data Security published on the internet 3 challenges about TTM (see [23]). On May $2^{\text {nd }}, 2000$, we managed to break the second challenge called TTM 2.1. The TTM 2.1 is a public key block cipher with plaintext block size 64 and ciphertext block size 100. It works on 8 bits finite field GF(256). The public key have been recovered with approximately 2000 queries to the "encryption oracle" available on the internet [23]. As mentioned in 2.4, its size is 214.5 Kbytes. Moreover it was broken in a simpler way that we described above. By iterative exploration of it's linearities, in 3 minutes on a PC we obtained the following plaintext which can be easily checked to be the exact solution to TTM 2.1. (note that the quotation marks are part of this plaintext):

"Tao TTP way BCKP of living hui mountain wen river moon love pt"

\section{The 'Kernel Attack' on MinRank and TPM}

In the present section we need the strategy of attack from 3.3 and use it with a new attack on $\operatorname{MinRank}(r)$, which works when $q^{r}$ is small enough.

Description of the Attack (notations are as in 3.3)

1. Choose $k$ random vectors $X^{\prime[1]}, \ldots, X^{\prime[k]}$ (with $k$ an integer depending on $n$ and $m$, that we define below). Since $\operatorname{dim} \operatorname{Ker}\left({ }^{\mathrm{t}} S A_{1} S\right)=n-\operatorname{Rank}\left({ }^{\mathrm{t}} S A_{1} S\right) \geq$ $n-r$, we have the simultaneous conditions $X^{\prime[i]} \in \operatorname{Ker}\left({ }^{\mathrm{t}} S A_{i} S\right)(1 \leq i \leq k)$ with a probability $\geq q^{-k r}$.

2. We suppose we have chosen a "good" set $\left\{X^{\prime[1]}, \ldots, X^{\prime[k]}\right\}$ of $k$ vectors (i.e. such that they all belong to $\left.\operatorname{Ker}\left({ }^{\mathrm{t}} S A_{1} S\right)\right)$. Then we can find an $m$-tuple $\left(\lambda_{1}, \ldots, \lambda_{m}\right)$ such that, for all $i, 1 \leq i \leq k,\left(\sum_{j=1}^{m} \lambda_{j} M_{j}\right)\left(X^{\prime[i]}\right)=0$. They are solution of a system of $k n$ linear equations in $m$ indeterminates. As a result, if we let $k=\left\lceil\frac{m}{n}\right\rceil$, the solution is essentially unique and can be easily found by Gaussian reduction. We thus obtain the two vector spaces $V_{0}=S^{-1}\left(K^{n-r} \times\{0\}^{r}\right)$ (corresponding to $x_{n-r+1}=\ldots=x_{n}=0$ ) and $W_{0}=S^{-1}\left(\{0\}^{n-r} \times K^{r}\right)$ (corresponding to $\left.x_{1}=\ldots=x_{n-r}=0\right)$.

3. The remaining part of the attack is exactly the same as in section 3.3

\section{Complexity of the Attack}

The complexity of the attack is easily computed: $\mathcal{O}\left(q^{\left\lceil\frac{m}{n}\right\rceil r} \cdot m^{3}\right)$. 


\section{Application to TTM}

In the particular case of TTM, we have $q=256, n=64, m=100$ and $r=2$.

We thus obtain an attack on TTM with complexity $\mathcal{O}\left(2^{52}\right)$.

Note: Compared to the $2^{28}$ of section 4.1 , this attack is slower, but it does not make use of any linearity of $y_{1}$ and $y_{2}$, so that it can also be used to break possible generalizations of TTM, with more general " $Q_{8}$ components" (see [4] for examples of $Q_{8}$ which provide non linear expressions for $y_{1}$ and $y_{2}$ over GF(2)).

\section{The 'Degeneracy Attack' on TPM Signature Schemes}

We describe here a general attack on TMP signature schemes (recall that such schemes are possible only for $u \leq r$ ), when $q^{u}$ is not too large. From the description of the attack, its complexity is easily seen to be $\mathcal{O}\left(q^{u} \cdot n^{6}\right)$. We use the same notations as in section 3.3. In particular, $m=n+u-r$.

1. We choose a random $m$-tuple $\left(\beta_{1}, \ldots, \beta_{m}\right) \in K^{m}$. With a probability $q^{-u-1}$, we can suppose that $\beta_{i} P_{i}$ is a degenerate quadratic polynomial (i.e. a quadratic polynomial which can be rewritten with fewer variables after a linear change of variables). The fact that a quadratic polynomial is degenerate can easily be detected: for instance by using its canonical form (see [18] for some other methods).

2. Suppose we have found a "good" $m$-tuple $\left(\beta_{1}, \ldots, \beta_{m}\right)$. Considering the new set of $(<n)$ variables for the quadratic form $\sum_{i=1}^{m} \beta_{i} P_{i}$, we deduce easily the vector space $W_{n-r}=S^{-1}\left(K^{n-r-1} \times\{0\} \times K^{r}\right)$.

3. Then we look for a $n$-tuple $\left(\alpha_{1}, \ldots, \alpha_{n}\right) \in K^{n}$ and a quadratic function $g^{n-r}$, such that:

$$
\sum_{i=1}^{m} \beta_{i} y_{i}^{\prime}=\sum_{i=1}^{n} \alpha_{i} x_{i}^{\prime}+g_{n-r}\left(x_{1}^{\prime}, \ldots, x_{n}^{\prime}\right)
$$

is true for any $\left(x_{1}^{\prime}, \ldots, x_{n}^{\prime}\right) \in W_{n-r}$. This can be done by Gaussian reduction. We thus obtain the vector space $V_{n-r}=S^{-1}\left(\{0\}^{n-r-1} \times K \times\{0\}^{r}\right)$ and the quadratic polynomial $g_{n-r}$.

4. The same principle can be repeated $n-r$ times, so as to obtain two sequences of vector spaces:

$$
\begin{gathered}
V_{n-r} \subseteq V_{n-r-1} \subseteq \ldots \subseteq V_{0} \\
W_{n-r} \supseteq W_{n-r-1} \supseteq \ldots \supseteq W_{0} .
\end{gathered}
$$

At the end, as in the attack described in section 3.3, we have completely determined the secret transformations $s$ and $t$, together with the secret functions $g_{i}$. As a result, this algorithm completely breaks the TPM family in signature mode (we recovered the secret key). 


\section{Conclusion}

We cryptanalysed a large class of cryptosystems TPM, that includes TTM as described by T.T. Moh [16]. They can be broken in polynomial time, as long as $r$ is fixed. The proposed TTM cryptosystem $\left[16\right.$ can be broken in $2^{28}$ due to linearities. Thus we could easily break the "TTM 2.1" challenge proposed by US Data Security in October 1997. Even if $Q_{8}$ was nonlinear, and since $r=2$, it is still broken in $2^{52}$ elementary operations for a 512-bit cryptosystem.

We also showed that signature schemes using TPM are insecure. There is very little hope that a secure triangular system will ever be proposed.

\section{References}

1. E.R. Berlekamp, R.J. McEliece, H.C.A. Van Tilborg, On the inherent intractability of certain coding problems, IEEE Transactions on Information Theory, IT-24(3), pp. 384-386, May 1978.

2. F. Chabaud, Asymptotic analysis of probabilistic algorithms for finding short codewords, in Proceedings of Eurocode'92, Udine, Italy, CISM Courses and lectures $\mathrm{n}^{\circ} 339$, Springer-Verlag, 1993, pp. 217-228.

3. K. Chen, A new identification algorithm, Cryptography Policy and Algorithms Conference, LNCS n 1029, Springer-Verlag, 1996.

4. C. Y. Chou, D. J. Guan, J. M. Chen, A systematic construction of a $Q_{2^{k}-\text { module }}$ in TTM, Preprint, October 1999. Available at http://www.usdsi.com/chou.ps

5. D. Coppersmith, S. Winograd, Matrix multiplication via arithmetic progressions, J. Symbolic Computation (1990), 9, pp. 251-280.

6. D. Coppersmith, J. Stern, S. Vaudenay, Attacks on the Birational Permutation Signature Schemes, in Advances in Cryptology, Proceedings of Crypto'93, LNCS $\mathrm{n}^{\circ} 773$, Springer-Verlag, 1993, pp. 435-443.

7. D. Coppersmith, J. Stern, S. Vaudenay, The Security of the Birational Permutation Signature Schemes, in Journal of Cryptology, 10(3), pp. 207-221, 1997.

8. N. Courtois, A. Shamir, J. Patarin, A. Klimov, Efficient Algorithms for solving Overdefined Systems of Multivariate Polynomial Equations, in Advances in Cryptology, Proceedings of EUROCRYPT'2000, LNCS n 1807 , Springer, 2000, pp. 392407.

9. N. Courtois: La sécurité des primitives cryptographiques basées sur les problèmes algébriques multivariables MQ, IP, MinRank, et HFE, PhD thesis, Paris 6 University, 26 September 2000, partly in English.

10. N. Courtois: The Minrank problem. MinRank, a new Zero-knowledge scheme based on the NP-complete problem. Presented at the rump session of Crypto 2000, available at http://www.minrank.org

11. H. Fell, W. Diffie, Analysis of a public key approach based on polynomial substitutions, in Advances in Cryptology, Proceedings of CRYPTO'85, LNCS n ${ }^{\circ} 218$, Springer-Verlag, 1985, pp. 340-349.

12. E.M. Gabidulin, Theory of codes with maximum rank distance, Problems of Information Transmission, 21:1-12, 1985.

13. S. Harari, A new authentication algorithm, in Coding Theory and Applications, LNCS n ${ }^{\circ} 388$, Springer, 1989, pp. 204-211. 
14. A. Kipnis, A. Shamir, Cryptanalysis of the HFE public key cryptosystem, in Advances in Cryptology, Proceedings of Crypto'99, LNCS n 1666, Springer, 1999, pp. $19-30$

15. T.T. Moh, A public key system with signature and master key functions, Communications in Algebra, 27(5), pp. 2207-2222, 1999. Available at http://www.usdsi.com/public.ps

16. T.T. Moh, A fast public key system with signature and master key functions, in Proceedings of CrypTEC'99, International Workshop on Cryptographic Techniques and E-commerce, Hong-Kong City University Press, pp. 63-69, July 1999. Available at http://www.usdsi.com/cryptec.ps

17. J. Patarin, Hidden Fields Equations (HFE) and Isomorphisms of Polynomials (IP): two new families of asymmetric algorithms, in Advances in Cryptology, Proceedings of EUROCRYPT'96, LNCS n ${ }^{\circ}$ 1070, Springer Verlag, 1996, pp. 33-48.

18. J. Patarin, L. Goubin, Asymmetric cryptography with S-Boxes, in Proceedings of ICICS'97, LNCS n 1334, Springer, 1997, pp. 369-380.

19. J.O. Shallit, G.S. Frandsen, J.F. Buss, The computational complexity of some problems of linear algebra, BRICS series report, Aarhus, Denmark, RS-96-33. Available at http://www.brics.dk/RS/96/33

20. A. Shamir, Efficient Signature Schemes based on Birational Permutations, in Advances in Cryptology, Proceedings of Crypto'93, LNCS n ${ }^{\circ} 773$, Springer-Verlag, 1993, pp. 1-12.

21. J. Stern, A new identification scheme based on syndrome decoding, in Advances in Cryptology, Proceedings of CRYPTO'93, LNCS n 773 , Springer-Verlag, 1993, pp. $13-21$.

22. J. Stern, F. Chabaud, The cryptographic security of the Syndrome Decoding problem for rank distance codes, in Advances in Cryptology, Proceedings of ASIACRYPT'96, LNCS n 1163 , Springer-Verlag, 1985, pp. 368-381.

23. The US Data Security Public-Key Contest, available at http://www.usdsi.com/contests.html

\section{Appendix: Actual Parameters for the TTM Cryptosystem}

Let $Q_{8}$ be the function defined by

$$
\begin{gathered}
Q_{8}\left(q_{1}, \ldots, q_{30}\right)=q_{1}^{8}+q_{29}^{4}+q_{30}^{2}+\left[q_{2}^{4}+q_{3}^{2} q_{8}^{2}+q_{4}^{2} q_{5}^{2}+q_{6}^{2} q_{12}^{2}+q_{7}^{2} q_{13}^{2}\right] \\
\times\left[q_{9}^{4}+\left(q_{10}^{2}+q_{14} q_{15}+q_{18} q_{19}+q_{20} q_{21}+q_{22} q_{24}\right)\left(q_{11}^{2}+q_{16} q_{17}+q_{23} q_{28}+q_{25} q_{26}+q_{13} q_{27}\right)\right] .
\end{gathered}
$$

However we obtain $Q_{8}\left(q_{1}, \ldots, q_{30}\right)=t_{19}^{2}$ as soon as we substitute the $q_{1 . .30}$ with:

\begin{tabular}{|c|c|c|c|}
\hline$q_{1}=t_{1}+t_{2} t_{6}$ & $q_{2}=t_{2}^{2}+t_{3} t_{7}$ & $q_{3}=t_{3}^{2}+t_{4} t_{10}$ & $q_{4}=t_{3} t_{5}$ \\
\hline$q_{5}=t_{3} t_{11}$ & $q_{6}=t_{4} t_{7}$ & $q_{7}=t_{4} t_{5}$ & $q_{8}=t_{7}^{2}+t_{5} t_{11}$ \\
\hline$q_{9}=t_{6}^{2}+t_{8} t_{9}$ & $q_{10}=t_{8}^{2}+t_{12} t_{13}$ & $q_{11}=t_{9}^{2}+t_{14} t_{15}$ & $q_{12}=t_{7} t_{10}$ \\
\hline$q_{13}=t_{10} t_{11}$ & $q_{14}=t_{12}^{2}+t_{7} t_{8}$ & $q_{15}=t_{13}^{2}+t_{11} t_{16}$ & $q_{16}=t_{14}^{2}+t_{10} t_{12}$ \\
\hline$q_{17}=t_{15}^{2}+t_{11} t_{17}$ & $q_{18}=t_{12} t_{16}$ & $q_{19}=t_{11} t_{12}$ & $q_{20}=t_{8} t_{13}$ \\
\hline$q_{21}=t_{7} t_{13}$ & $q_{22}=t_{8} t_{16}$ & $q_{23}=t_{14} t_{17}$ & $q_{24}=t_{7} t_{11}$ \\
\hline$q_{25}=t_{12} t_{15}$ & $q_{26}=t_{10} t_{15}$ & $q_{27}=t_{12} t_{17}$ & $q_{28}=t_{11} t_{14}$ \\
\hline$q_{29}=t_{18}+t_{1}^{2}$ & $q_{30}=t_{19}+t_{18}^{2}$ & \multicolumn{1}{|l}{}
\end{tabular}


We put $n=64, v=36$, and we consider the $t_{i}=t_{i}\left(u_{1}, \ldots, u_{19}\right)(1 \leq i \leq 19)$ as randomly chosen linear forms (i.e. homogeneous polynomials of degree one in $\left.u_{1}, \ldots, u_{19}\right)$, satisfying the following conditions:

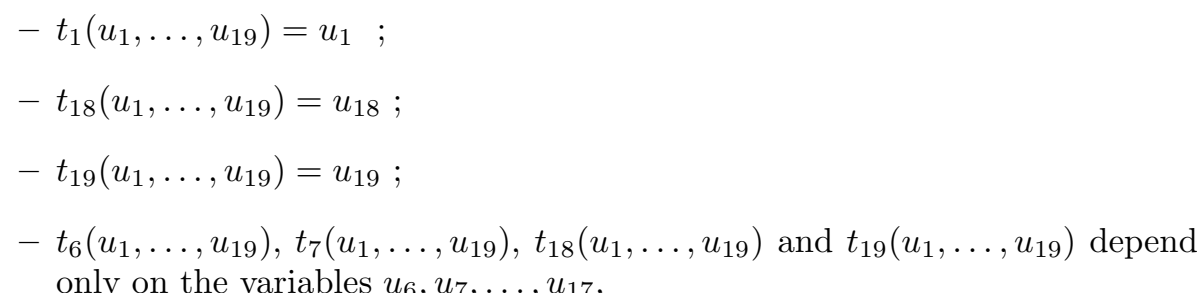
only on the variables $u_{6}, u_{7}, \ldots, u_{17}$,

We thus obtain polynomials $q_{i}=q_{i}\left(u_{1}, \ldots, u_{19}\right)(1 \leq i \leq 30)$ of degree two in $u_{1}, \ldots, u_{19}$. Finally, we choose:

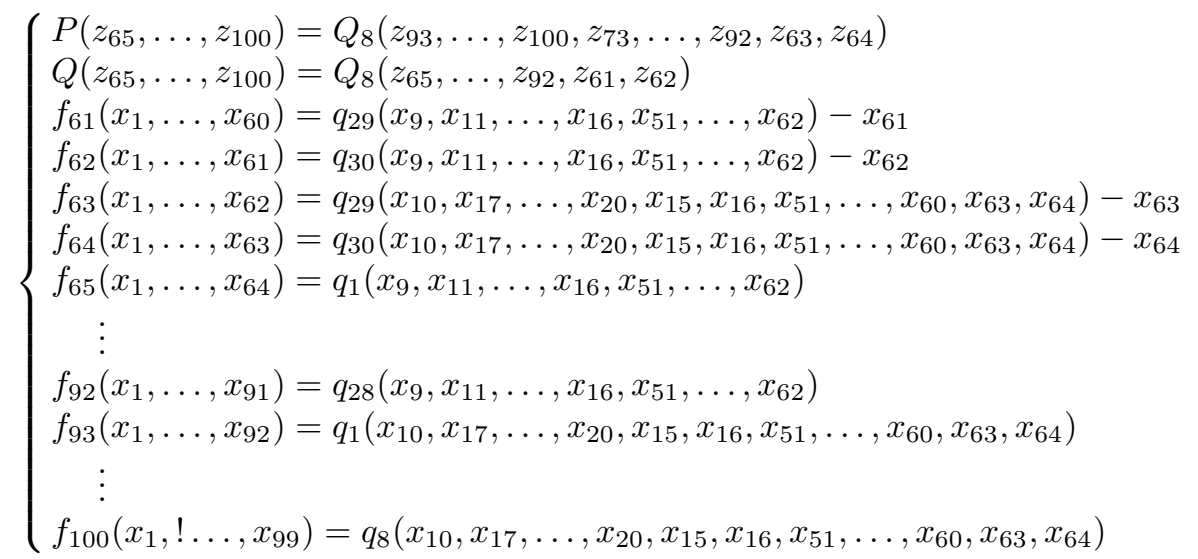

and randomly chosen quadratic forms for $f_{i}(2 \leq i \leq 60)$.

Let us denote $\theta: K^{64} \rightarrow K^{100}$ the function defined by

$$
\theta\left(x_{1}, \ldots, x_{64}\right)=\left(x_{1}, \ldots, x_{64}, 0, \ldots, 0\right)
$$

Hence $\left(x_{1}, \ldots, x_{64}\right) \mapsto\left(y_{1}, \ldots, y_{100}\right)=\Phi_{3} \circ \Phi_{2} \circ \theta\left(x_{1}, \ldots, x_{64}\right)$ is given by the following system: 


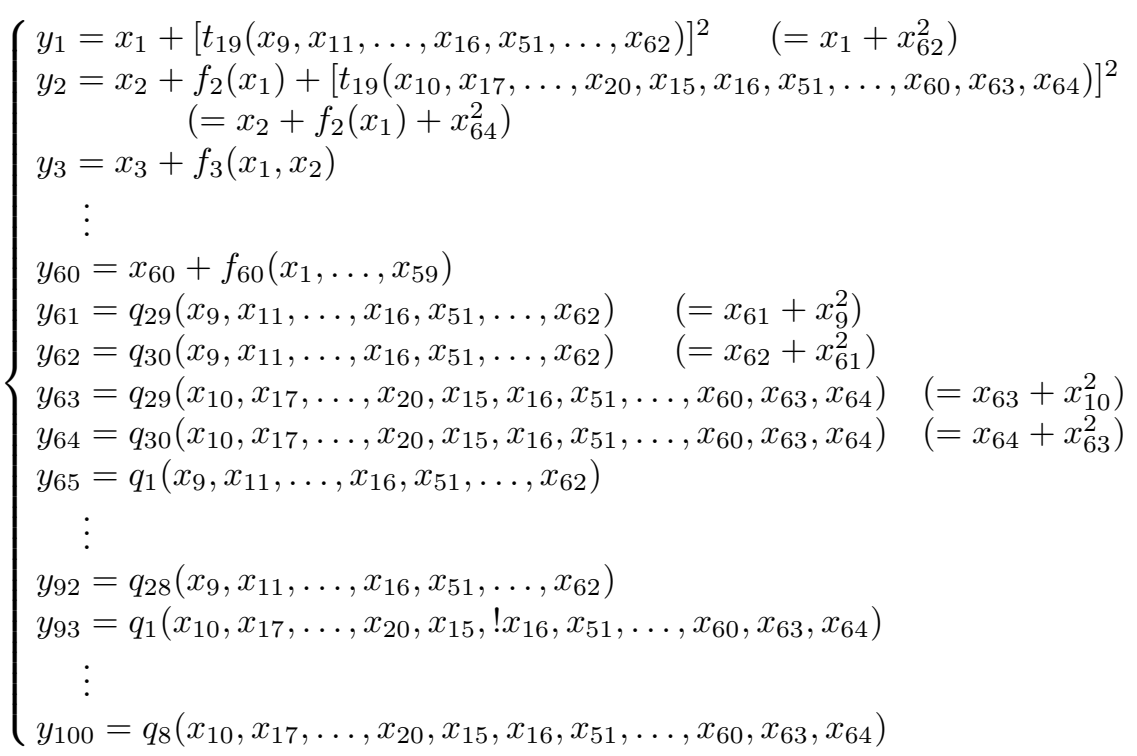

\section{The Public Key}

The user selects a random invertible affine transformation $\Phi_{1}: K^{64} \rightarrow K^{64}$, and a random invertible affine transformation $\Phi_{4}: K^{100} \rightarrow K^{100}$, such that the function $F=\Phi_{4} \circ \Phi_{3} \circ \Phi_{2} \circ \theta \circ \Phi_{1}$ satisfies

$$
F(0, \ldots, 0)=(0, \ldots, 0) .
$$

By construction of $F$, if we denote $\left(y_{1}^{\prime}, \ldots, y_{100}^{\prime}\right)=F\left(x_{1}^{\prime}, \ldots, x_{64}^{\prime}\right)$, then we have an explicit set $\left\{P_{1}, \ldots, P_{100}\right\}$ of 100 quadratic polynomials in 64 variables, such that:

$$
\left\{\begin{array}{c}
y_{1}^{\prime}=P_{1}\left(x_{1}^{\prime}, \ldots, x_{64}^{\prime}\right) \\
\quad \vdots \\
y_{100}^{\prime}=P_{100}\left(x_{1}^{\prime}, \ldots, x_{64}^{\prime}\right)
\end{array}\right.
$$

This set of 100 polynomials constitutes the public key of the TTM cryptosystem.

\section{Encrypting a Message}

Given a plaintext $\left(x_{1}^{\prime}, \ldots, x_{64}^{\prime}\right) \in K^{64}$, the sender computes $y_{i}^{\prime}=P_{i}\left(x_{1}^{\prime}, \ldots, x_{64}^{\prime}\right)$ for $1 \leq i \leq 100$ (thanks to the public key) and sends the ciphertext $\left(y_{1}^{\prime}, \ldots, y_{100}^{\prime}\right)$.

\section{Decrypting a Message}

Given a ciphertext $\left(y_{1}^{\prime}, \ldots, y_{100}^{\prime}\right) \in K^{100}$, the legitimate receiver recovers the plaintext by:

$$
\left(x_{1}^{\prime}, \ldots, x_{64}^{\prime}\right)=\Phi_{1}{ }^{-1} \circ \pi \circ \Phi_{2}^{-1} \circ \Phi_{3}{ }^{-1} \circ \Phi_{3}{ }^{-1} \circ \Phi_{4}{ }^{-1}\left(y_{1}^{\prime}, \ldots, y_{100}^{\prime}\right)
$$

with $\pi: K^{100} \mapsto K^{64}$ defined by $\pi\left(x_{1}, \ldots, x_{100}\right)=\left(x_{1}, \ldots, x_{64}\right)$ and thus satisfies $\pi \circ \theta=$ Id. 